\title{
Probing Chiral Nanoparticles and Surfaces by Infrared Spectroscopy
}

\author{
Cyrille Gautiera, Marco Bieria, Igor Dolamic ${ }^{a}$, Silvia Angelonib, Julien Boudonª, and \\ Thomas Bürgi*a
}

\begin{abstract}
Chiral metal surfaces and nanoparticles have the potential to be used for the selective production, the resolution and the detection of enantiomers of a chiral compound, which renders them highly attractive in view of the tremendous consequences of homochirality on earth. Their capability to distinguish between enantiomers of a chemical compound relies on their structure and the ability to form intermolecular interactions. However, molecular-level understanding of the interactions that are at the origin of enantiodiscrimination is lagging behind due to the lack of powerful experimental techniques that are able to spot these interactions selectively with high sensitivity. In this article two techniques based on infrared spectroscopy are presented that are able to selectively target the chiral properties of nanoparticles and interfaces. These are the combination of attenuated total reflection infrared (ATR-IR) with modulation excitation spectroscopy (MES) to probe enantiodiscriminating interactions at chiral solid-liquid interfaces and vibrational circular dichroism (VCD), which is used to probe the structure of chirally modified metal nanoparticles.
\end{abstract}

Keywords: Chiral surfaces · Enantiodiscrimination · Nanoparticles · SAMs · Vibrational circular dichroism

\section{Introduction}

Chirality - the left- or right-handedness of objects - is all around us. On the molecular scale chirality has dramatic consequences. Whenever a chiral compound interacts with a metabolism, the two enantiomers can show distinctly different behavior. On the nanometer-scale the control of chirality is critical for the preparation of catalysts, for the production of chiral compounds and for

\footnotetext{
${ }^{\star}$ Correspondence: Prof. Dr. T. Bürgi ${ }^{\mathrm{a}}$

Tel.: +41327182412

Fax: +41327182511

E-Mail: thomas.burgi@unine.ch

aUniversité de Neuchâtel

Institut de Microtechnique

Rue Emile-Argand 11

Case Postale 158

$\mathrm{CH}-2009$ Neuchâtel

${ }^{b}$ Centre Suisse d'Electronique

et de Microtechnique SA (CSEM)

Rue Jaquet-Droz 1

P.O. Box

CH-2002 Neuchâtel
}

their resolution. This is particularly important for the pharmaceutical industry, but is also relevant to the agrochemical and flavor and fragrances sectors. A key question in this respect is: How is chirality expressed from the molecular scale to the nanometer scale?

Recently this question has stimulated interest in the surface science community and has led to numerous reports on chiral or chirally modified metal surfaces. Chemists tend to associate chirality with organic molecules, inorganic salts and biological materials but not necessarily with metals and surfaces. However, metal surfaces can indeed be chiral in different ways. Some high Miller index surfaces of metals such as for example $\operatorname{Ag}(643)$ are intrinsically or naturally chiral [1][2]. A different type of chirality is obtained through adsorption and long-range ordering of molecules on non-chiral metal surfaces [3], if the pattern formed by the adsorbate destroys the symmetry of the underlying metal surface. The adsorbate itself does not even have to be chiral in this case resulting in a racemic mixture of domains on the surface with opposite chirality [4]. Furthermore, adsorption of a chiral molecule [5-8] creates a locally chiral environment near the metal surface. This may also induce a slight distortion of the metal surface atoms involved in the adsorbate complex toward a chiral arrangement, i.e. the creation of a chiral 'footprint'
[9]. The chirality of an adsorbate may also be transferred onto the electronic structure of the non-chiral metal [10].

Monolayer protected metal nanoparticles (MPNs) [11] can be viewed as the nanometer-sized analogues of adsorbatecovered flat metal surfaces and in principle the same types of chirality could arise as discussed above for extended metal surfaces. Due to their solubility, i.e. the possibility to prepare stable dispersions, such particles can be investigated by chiroptical techniques such as circular dichroism (CD) [12] and vibrational circular dichroism (VCD) spectroscopy [13]. The former technique probes electronic transitions, which may be located in the metal core, whereas the latter is sensitive towards vibrations of molecules within the protective monolayer enclosing the nanoparticle. For both methods the observed signals are intimately related to the chirality of the sample. If the sample is nonchiral or racemic no signals are observed. VCD is sensitive towards conformation and has been used in the past for structure determination of dissolved molecules. We have shown recently that the method can also be used to study the conformation of chiral molecules adsorbed on metal nanoparticles [14], which is interesting also because the structure of the adsorbed molecules determine important properties of the nanoparticles such as molecular recognition and organization. 
A valuable property of chiral surfaces and nanoparticles is their ability to discriminate between enantiomers, which is crucial for heterogeneous enantioselective catalysis [15][16], separation [17] and sensing [18] of enantiomers. Today not much is known about the mechanisms of enantiodiscrimination, i.e. the relevant intermolecular interactions between surface (selector) and analyte molecule (selectand) because most of the available experimental methods merely quantify enantiodiscrimination without giving direct molecularlevel insight.

A technique to probe enantiodiscrimination at interfaces should ideally combine (surface) sensitivity with selectivity for the chiral information. The combination of both attributes is a real challenge. Infrared (IR) spectroscopy is a well-established tool for the investigation of interfaces. However, conventional IR spectroscopy has the disadvantage that non-specific interactions also give rise to signals. In order to overcome these problems we have combined attenuated total reflection infrared (ATR-IR) [19] with modulation excitation spectroscopy (MES) [20] to a tool that can be used to study enantiodiscrimination at chiral solid -liquid interfaces. In this article we describe the application of ATR-IR/MES for the study of self-assembled monolayers of chiral thiols on gold surfaces as well as the characterization of their nanometer-size analogues by chiroptical techniques.

\section{Chiral Gold Nanoparticles}

Monolayer protected gold nanoparticles (MPNs) can be prepared by reducing an $\mathrm{Au}(\mathrm{I})$-thiol polymer. The latter is spontaneously formed by mixing an Au(III) salt with a thiol. During reduction of the polymer the particles form according to a nucleation, growth passivation mechanism. We have used chiral thiols such as $\mathrm{N}$-acetyl-cysteine and $\mathrm{N}$-isobutyryl-cysteine (Scheme) for the preparation of water-soluble gold particles. The size of the latter is typically very small, in the order of $2 \mathrm{~nm}$ gold core diameters or

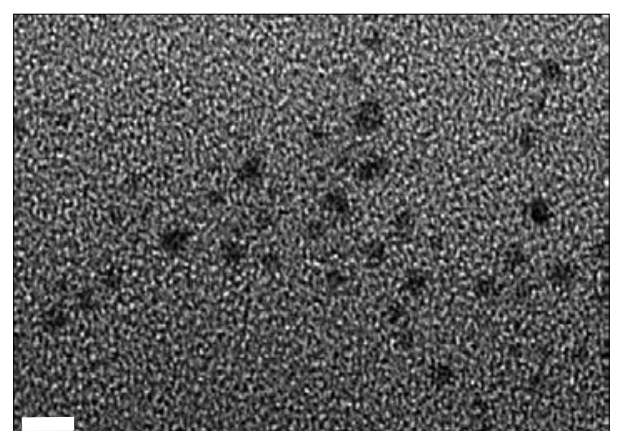

\section{$5 \mathrm{~nm}$}

Fig. 1. Transmission electron micrograph (TEM) of gold nanoparticles covered with $\mathrm{N}$-acetyl-Lcysteine.

less, as can be seen from the transmission electron micrograph in Fig. 1.

Fig. 2 shows IR and VCD spectra of Nisobutyryl-D/L-cysteine MPNs dissolved in $\mathrm{D}_{2} \mathrm{O} / \mathrm{NaOH}$ solution [21]. The IR spectra of the two MPN samples are identical but the VCD spectra show a mirror image relationship for the nanoparticles covered with the enantiomers of $\mathrm{N}$-isobutyryl-cysteine. Note that the measurements were performed on the as-prepared samples, which are not mono-disperse. The IR and VCD spectra are however a local property of the individual adsorbed molecules and therefore the size of the particles has only a minor influence.

The structural information contained in the VCD spectra has to be extracted through a comparison with calculations. More precisely, VCD spectra for different conformations of the adsorbed molecule have to be calculated and compared with experiment. Fig. 3 shows some conformers of deprotonated $\mathrm{N}$-isobutyryl-L-cysteine adsorbed on an $\mathrm{Au}_{8}$ cluster that were detected and Fig. 4 shows the corresponding calculated VCD spectra. The primary anchoring of the molecule to the cluster is through the sulfur atom in a bridge site, i.e. in-between two $\mathrm{Au}$ atoms, in agreement with recent periodic density functional theory (DFT) calculations for cysteine on $\mathrm{Au}(111)$ [22].

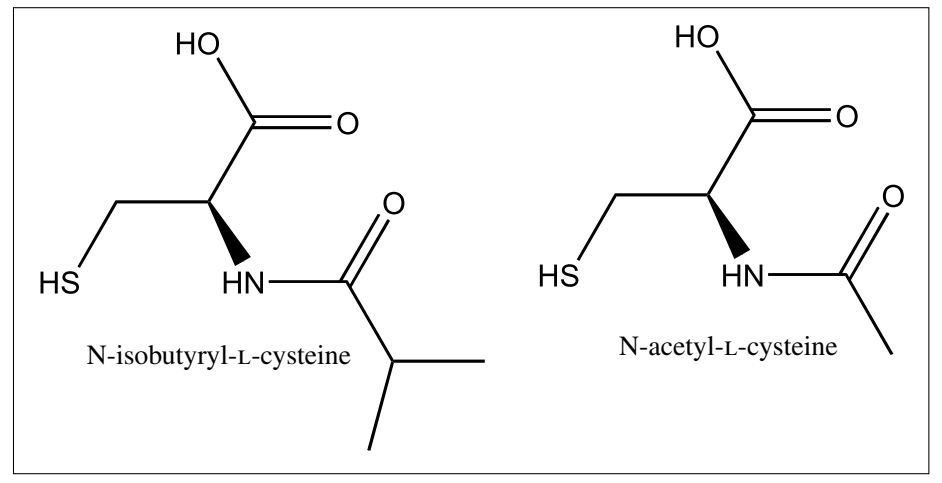

Scheme. N-isobutyryl-L-cysteine and N-acetyl-L-cysteine

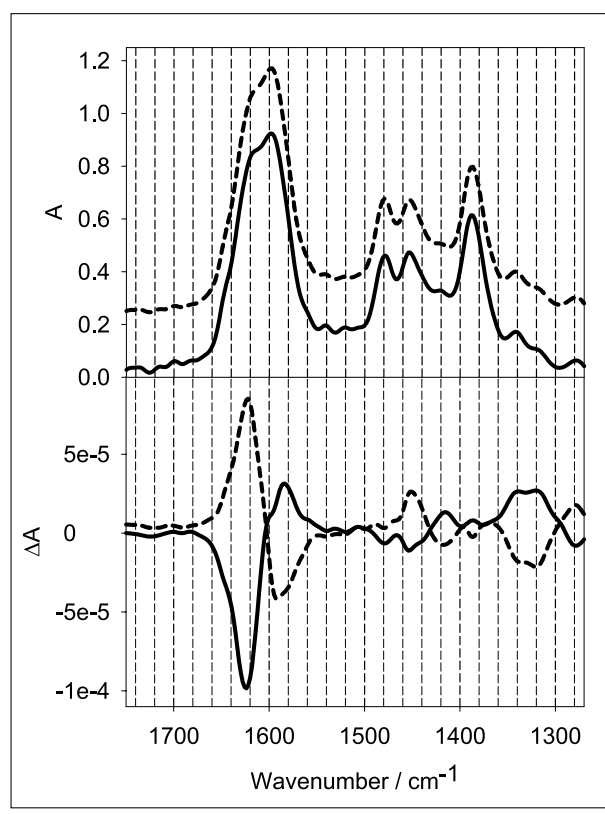

Fig. 2. Infrared (top) and VCD (bottom) spectra of $\mathrm{N}$-isobutyryl-cysteine protected gold nanoparticles in $\mathrm{D}_{2} \mathrm{O} / \mathrm{NaOH}$. Solutions were made from 7 $\mathrm{mg}$ sample in $50 \mu \mathrm{l}$ solution. The dashed (solid) lines correspond to the spectra of the particles covered by the L-enantiomer (D-enantiomer). The IR spectrum of the particles covered with $\mathrm{N}$-isobutyryl-L-cysteine was offset for clarity. Reprinted with permission from [21]. Copyright (2005) American Chemical Society.

The most important conformational degrees of freedom of the molecule are the rotations around the three single bonds involving the asymmetric $\mathrm{C}$ atom (two $\mathrm{C}-\mathrm{C}$ bonds and one $\mathrm{C}-\mathrm{N}$ bond). The isopropyl group can adopt several positions with respect to the rest of the molecule (compare conformations a-c in Fig. 3). However, we have noticed that the rotation of the isopropyl group has only a minor influence on the VCD spectra, as is obvious from Fig. 4 , spectra a-c.

The more stable conformers interact with the $\mathrm{Au}_{8}$ cluster not only via the sulfur atom but also via the carboxylate. The two most stable conformers a and $\mathrm{f}$ differ only by the position of the asymmetric carbon atom within the seven-membered ring formed by the molecule and the $\mathrm{Au}_{8}$ cluster (-Au-S-C-C-C-O-Au-). Still, their VCD spectra are distinctly different. For the most stable conformer $\mathrm{f}$ almost all prominent VCD bands are negative, in contrast to experiment (see Fig. 2). The VCD spectrum of conformer d exhibits two overlapping positive bands for the amide I and $v_{\text {as }}\left(-\mathrm{COO}^{-}\right)$ vibrations (calculated slightly above 1700 $\mathrm{cm}^{-1}$ ) in contrast to experiment. Conformers a (and also the related conformers $b$ and c) and e exhibit the correct sign for the amide $\mathrm{I}$ (positive) and $\mathrm{v}_{\mathrm{as}}\left(-\mathrm{COO}^{-}\right)$(negative) vibrations. Based on the calculated energy conformer e is unlikely. The VCD spectrum of conformer a (also b and c) fits 


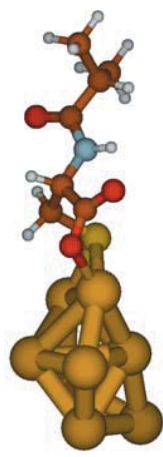

a) $1.7 \mathrm{kcal} / \mathrm{mol}$

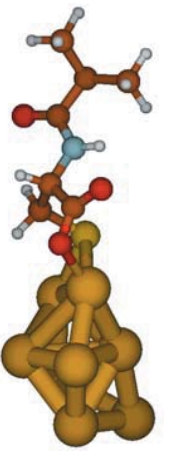

b) $2.8 \mathrm{kcal} / \mathrm{mol}$

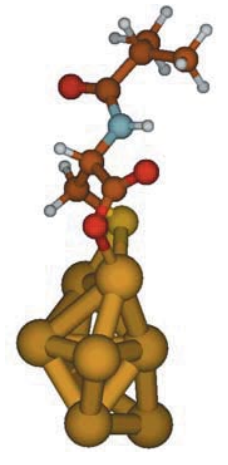

c) $2.8 \mathrm{kcal} / \mathrm{mol}$

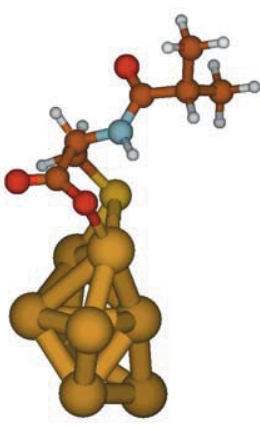

d) $3.5 \mathrm{kcal} / \mathrm{mol}$

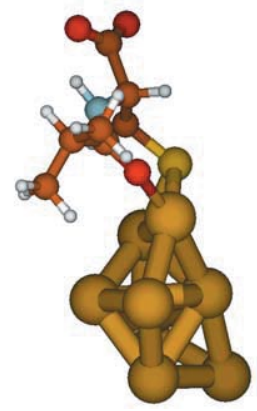

e) $13.5 \mathrm{kcal} / \mathrm{mol}$

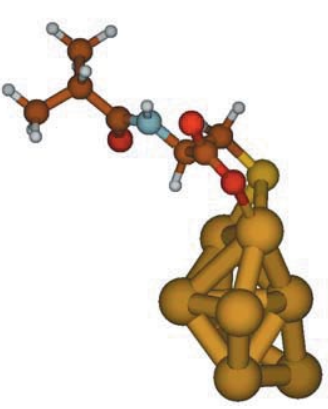

f) $0.0 \mathrm{kcal} / \mathrm{mol}$
Fig. 3. Calculated conformers of deprotonated $\mathrm{N}$-isobutyryl-L-cysteine on an $\mathrm{Au}_{8}$ cluster. The numbers indicate the calculated relative stability with respect to the most stable conformer $f$. The calculations were performed at the B3PW91 level using a 6-31G(d,p) (LanL2DZ for Au) basis set. Reprinted with permission from [21]. Copyright (2005) American Chemical Society.

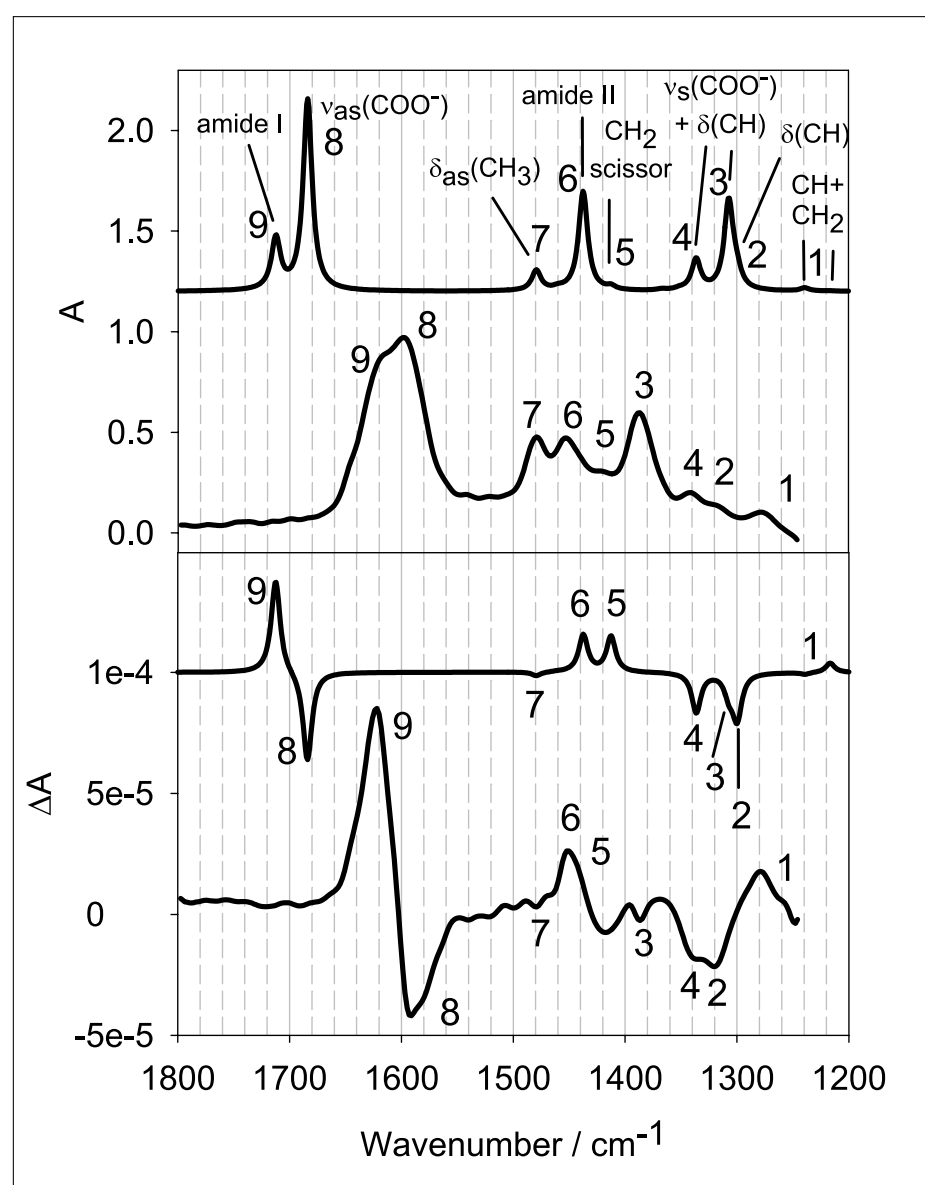

Fig. 5. Comparison between calculated and experimental IR and VCD spectra of $\mathrm{N}$-isobutyryl-L-cysteine on gold. The calculated spectra are shown for conformer a in Fig. 3. Corresponding bands are numbered and the assignment is given in the upper part of the Figure. Reprinted with permission from [21]. Copyright (2005) American Chemical Society.

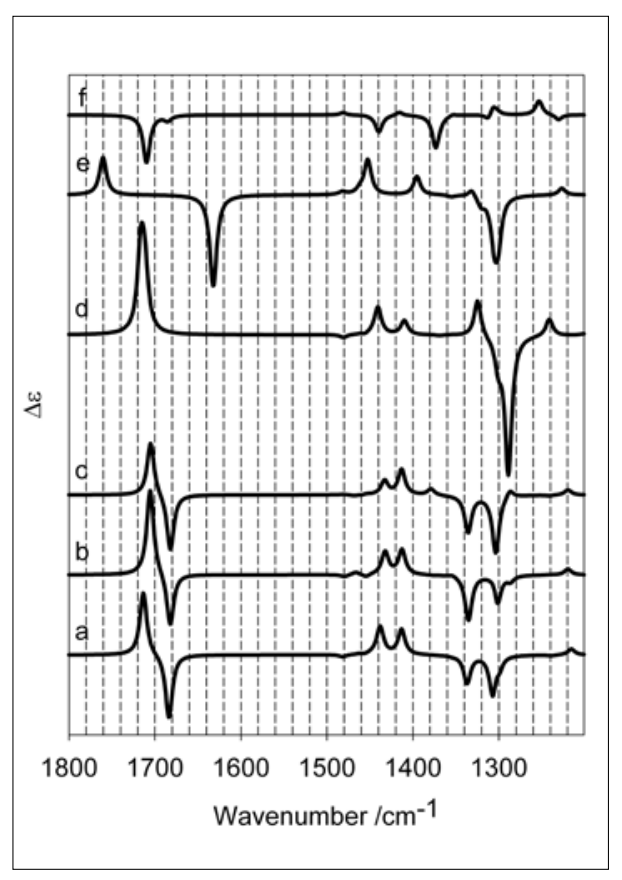

Fig. 4. Calculated VCD spectra for different conformers of $\mathrm{N}$-isobutyryl-L-cysteine adsorbed on an $\mathrm{Au}_{8}$ cluster. The letters correspond to the structures shown in Fig. 3. Reprinted with permission from [21]. Copyright (2005) American Chemical Society. qualitatively well with experiment. The calculated IR and VCD spectra of conformer a are compared with the experimental spectra for $\mathrm{N}$-isobutyryl-L-cysteine protected gold MPNs in Fig. 5. Most importantly, the investigation shows that VCD spectroscopy is a powerful tool for structure determination of chiral molecules adsorbed on small metal particles. At this point we shall mention some challenges of VCD applied to nanoparticles. The signals are small, as is observed in general for VCD, which may require long measurements (hours) to reduce noise. In addition the measurements require a relatively large amount of sample (1-5 mg). It should also be mentioned that apart from the work reviewed here, no VCD of nanoparticles has been reported before, which makes a critical review difficult at the present time.

The conformation of $\mathrm{N}$-isobutyryl-Lcysteine adsorbed on gold nanoparticles emerging from the VCD investigations resembles closely the one determined for $\mathrm{N}$ acetyl-L-cysteine on gold surfaces based on attenuated total reflection infrared spectroscopy [7][23]. In the latter study the orientation of the molecule on the gold surface was determined from the orientation of the tran- sition dipole moments for different vibrations. A similar structure was also proposed for cysteine adsorbed on $\mathrm{Cu}$ surfaces [24].

Due to the carboxylic acid group of $\mathrm{N}$-isobutyryl-cysteine the corresponding MPNs are charged, which can be used for their separation according to size and charge in a gel electrophoresis. Fig. 6 shows a photograph of a gel after separation. Eight compounds could be separated and were numbered 1-8 according to increasing particle size. TEM analysis reveals that compound 2 (the smallest compound observable by TEM) is characterized by a particle size of about $0.7 \mathrm{~nm}$, whereas compound 7 (the largest well-defined compound) is characterized by $1.3 \mathrm{~nm}$ particles. The color change with the size of the particles is a direct consequence of a quantum size effect. This is also evident from the UV-vis spectra (not shown), which show a rich structure. The appearance of discrete bands in the gel electrophoresis furthermore reveals the presence of 'magic number' compounds, i.e. the high relative abundance of compounds with well-defined composition (number of $\mathrm{Au}$ atoms and ligands).

The isolated compounds show optical activity in metal-based transitions. CD 


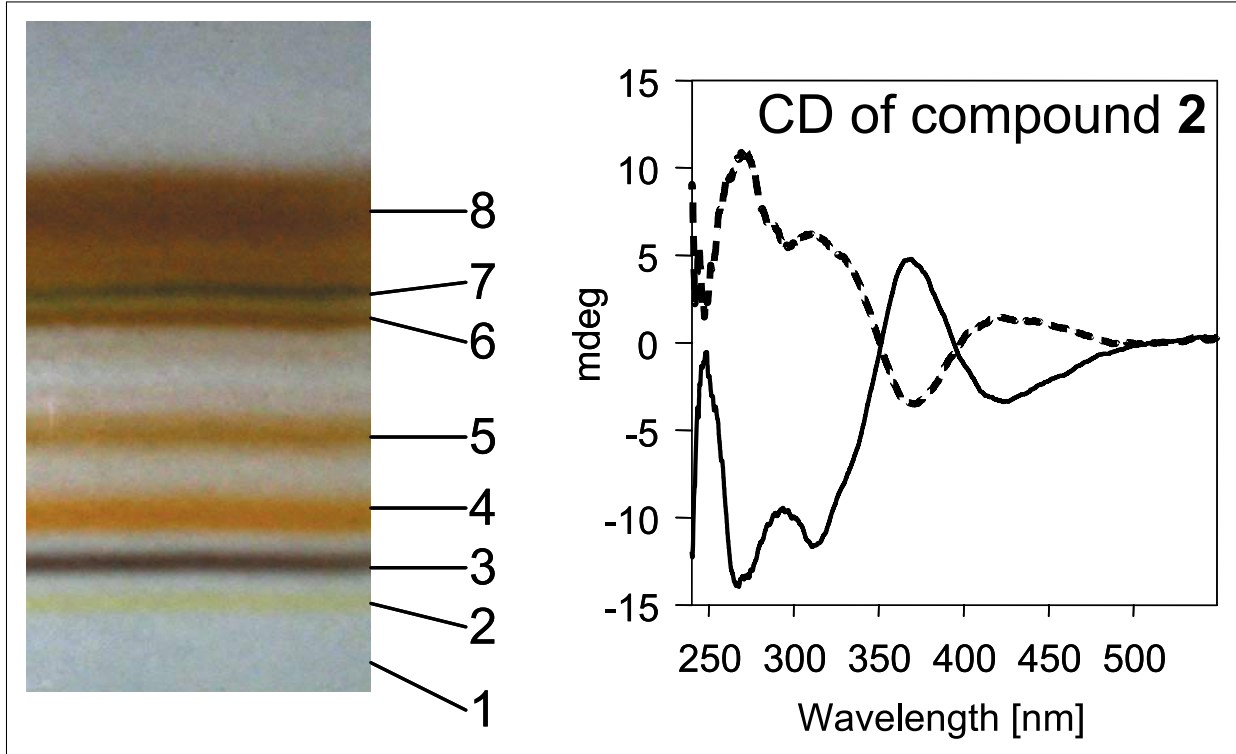

Fig. 6. Left: Polyacrylamide gel electrophoresis (PAGE) separation of $\mathrm{N}$-isobutyryl-L-cysteine protected gold particles. The separation was performed for $17 \mathrm{~h}$ at $150 \mathrm{~V}$. The separated compounds are numbered from 1-8 according to their decreasing electrophoretic mobility. Compound $\mathbf{1}$ is only visible under UV irradiation. Right: CD spectra of separated compound $\mathbf{2}$ of gold particles covered with $\mathrm{N}$ isobutyryl-L-cysteine (dashed) and $\mathrm{N}$-isobutyryl-L-cysteine (solid line). Adapted with permission from [21]. Copyright (2005) American Chemical Society.

spectra of compound 2 are shown in Fig. 6. The two spectra correspond to MPNs that are covered with the two enantiomers of $\mathrm{N}$ isobutyryl-cysteine. The CD spectra of the two samples show a mirror image relationship. Note that the corresponding spectra of $\mathrm{N}$-isobutyryl-cysteine exhibit optical activity only below $300 \mathrm{~nm}$. The observation of CD signals associated with electronic transitions located in the metal core shows that the electronic structure of the gold cores is chiral. The key question emerging from this study is whether the structure of the metal core is intrinsically chiral or whether the CD signals in the metallic transitions are induced by the chiral ligands adsorbed on a non-chiral metal core. Due to the lack of direct structural information this question remains open. Theoretical calculations indicate that bare, small metal particles such as $\mathrm{Au}_{28}$ prefer low symmetry chiral over high symmetry achiral structures [25]. The chiral arrangement of the ligands or the chiral structure of the ligand itself could also induce $\mathrm{CD}$ in the metal-based transitions. Induced $\mathrm{CD}$ occurs when transition dipole moments of optically active electronic transitions couple to transition dipole moments of another species, which may be achiral. Using a dissymmetrically-perturbed particle-in-a-box model it has been shown recently that $C D$ signals can be induced by chiral adsorbates on symmetric particles [26]. The CD signals observed for the chiral gold particles are relatively strong. The magnitude of the anisotropy factor $g=\Delta \varepsilon / \varepsilon$ is in the order of $1 / 1000$ and thus similar to those reported for intrinsically chiral fullerenes [27] and fullerenes with chiral functionalization patterns [28]. Fullerenes with an attached optically active group but with non-chiral functionalization pattern showed considerably weaker CD response [28]. This comparison would point more towards an intrinsically chiral metal core (or chiral 'footprint') or a chiral adsorbate pattern and possibly the characteristic double point adsorption of $\mathrm{N}$-isobutyryl-cysteine as elucidated by VCD, plays a crucial role in bestowing chirality to the metal particle.

\section{Enantiodiscrimination at Chiral Self-assembled Monolayers}

Chiral nanoparticles and the corresponding self-assembled monolayers can distinguish between the enantiomers of a probe molecule. In order to gain molecular-level insight into the mechanism of enantioselection, which is a prerequisite for the rational design of enantiodiscriminating systems, we have proposed a method that combines attenuated total reflection infrared (ATR-IR) with modulation excitation spectroscopy (MES) [20] as schematically depicted in Fig. 7. An internal reflection element (IRE) for ATR-IR is first coated with a thin gold layer. The ATR-IR technique allows solid-liquid interfaces to be probed in situ via an evanescent field. The self-assembly of a chiral thiol on the gold surface is then performed and followed by ATR-IR to prepare a chiral interface. Solutions of the two enantiomers of the chiral probe molecule (selectand) are then periodically flowed over the chiral self-assembled monolayer (SAM, selector). In this way the concentration of the two enantiomers is by turns high and low, respectively, while the total concentration stays constant throughout the modulation experiment (see concentration profile on the left in Fig. 7). During the modulation ATR-IR spectra are recorded. These time-resolved spectra are subsequently demodulated, i.e. transformed into phase-resolved or demodulated spectra (phase sensitive detection) [20]. Only the periodically varying signals are thus detected and therefore no dissolved species are observed, because the IR spectra of the dissolved enantiomers are identical. If the enantiomers adsorb on a non-chiral surface or a non-chiral site their spectra remain identical and therefore unspecific interactions are suppressed. Adsorption of the enantiomers on chiral sites that can distinguish between them leads to diastereomeric complexes (selector-selectand complexes) with (slightly) different IR spectra, which are therefore selectively observable by the absolute configuration modulation technique. The advantages of the method has been demonstrated by studying the interaction between chiral molecules and chiral stationary phases used in chromatography [29][30]. More recently the technique was used to study the enantiodiscrimination of a chiral SAM of L-glutathione (GSH, $\gamma$ glu-cys-gly) on gold. The latter SAM could quite efficiently discriminate between the enantiomers of proline. In the following the interaction between SAMs of $\mathrm{N}$-acetyl-L-cysteine (NAC, Scheme) on gold and proline are described.

ATR-IR spectra of the interacting species, NAC adsorbed on gold (selector, bold solid line) and dissolved proline (selectand, thin solid line) are shown in Fig. 8, bottom. The most prominent vibrational modes for NAC are the $v(\mathrm{COOH})$ mode at $1727 \mathrm{~cm}^{-1}$, amide I at $1661 \mathrm{~cm}^{-1}$ and amide II at $1539 \mathrm{~cm}^{-1}$. The proline ATR-IR spectrum is dominated by the $v_{\text {as }}\left(\mathrm{COO}^{-}\right)$ vibrational mode at $1637 \mathrm{~cm}^{-1}$ with the $\delta_{\mathrm{s}}\left(\mathrm{NH}_{2}\right)$ scissoring mode appearing as a shoulder at $1570 \mathrm{~cm}^{-1}$. On top in Fig. 8 two-phase-resolved, i.e. demodulated spectra are presented (label '(D) vs. (L)') that refer to absolute configuration modulation experiments performed on different days using different gold surfaces (gold coated Ge IREs). These phase-resolved ATR-IR spectra highlight the differences in intermolecular interactions between the two proline enantiomers and the NAC SAM. The spectra show a rather complex pattern with sharp positive and negative bands. Although the signals are considerably above the noise level (which is about $5 \times 10^{-6}$ in the spectral region around $1700 \mathrm{~cm}^{-1}$ ) the overall strength of the ATR-IR signals reveals a rather weak enantiodiscrimination between the NAC SAM and proline. The dispersive bands in demodulated spectra arise due to slight frequency shifts induced by the stimulation, i.e. the change in abso- 


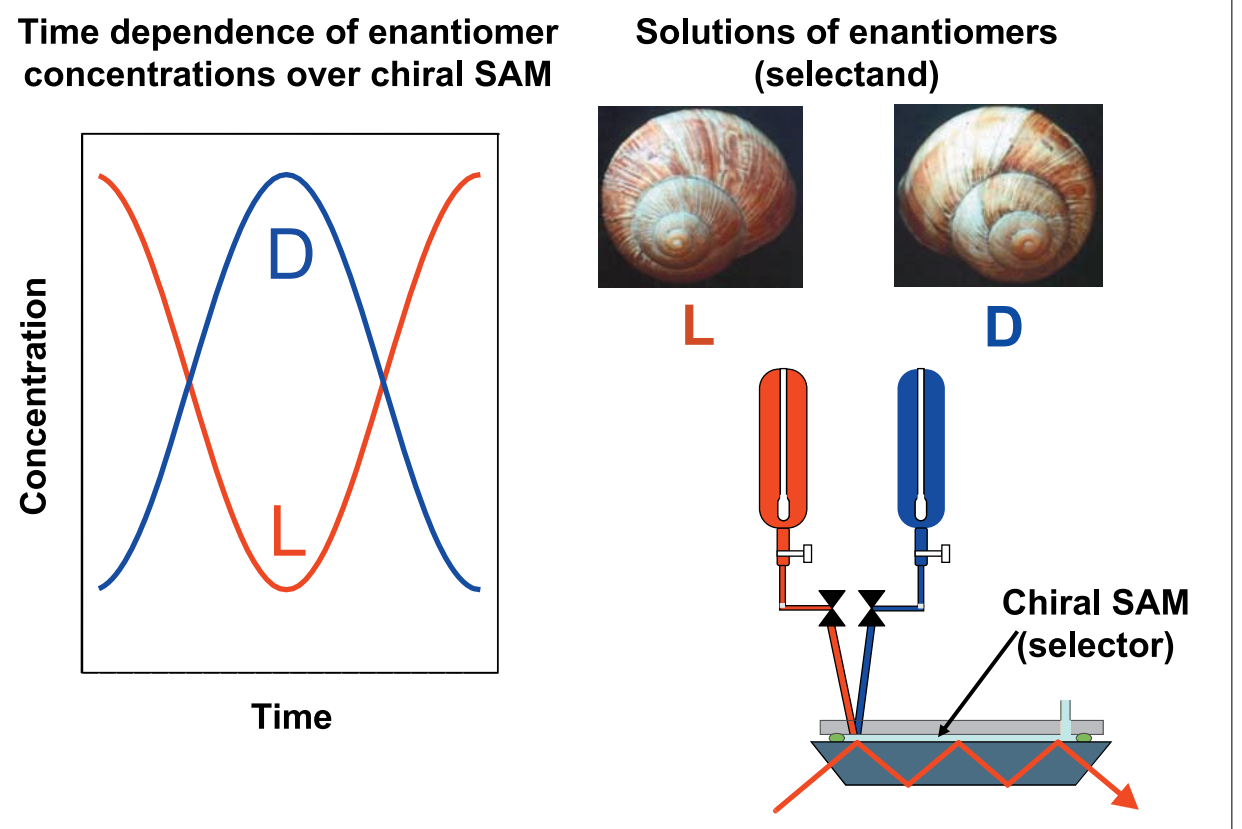

Fig. 7. Principle of absolute configuration modulation combined with attenuated total reflection IR spectroscopy for investigating the interaction of chiral molecules with chiral surfaces. Left: Schematic representation of time-dependent concentration of individual enantiomers in an absolute configuration modulation excitation experiment. The total concentration of both enantiomers remains constant. The stimulation is sinusoidal in the depicted example, which is however not a necessary requirement. Right: Two bubble tanks containing the solutions of the two enantiomers are connected to the flowthrough cell. The solutions can be alternately flowed over the sample ('modulated') by switching the two computer controlled pneumatically actuated Teflon valves. During the 'modulation' ATR-IR spectra are measured.
The calculated IR spectra for the NACD-proline complex, denoted by '(D)', and for the NAC-L-proline complex, denoted by '(L)', together with the corresponding difference spectrum (bold solid line, '(L)-(D)' is displayed in Fig. 9, bottom. At first glance the calculated spectra of the (D) and $(\mathrm{L})$ complexes seem to be identical but the resulting difference spectrum, scaled by a factor 5, clearly reveals significant dispersive bands that originate from slight frequency shifts of vibrational bands in the order of one to several wavenumbers. This difference spectrum is directly comparable to the experimental spectra shown in Fig. 8 , top. The calculations are in qualitative agreement with experiment. In particular, the observed dispersive line pattern between 1700 and $1550 \mathrm{~cm}^{-1}$ is very well reproduced by the calculations. The agreement between experiment and theory finally allows the conclusion that enantiodiscrimination is dominated by a 'one-point' electrostatic interaction between proline $\left(\mathrm{NH}_{2}^{+}\right)$and NAC $\left(\mathrm{COO}^{-}\right)$. A 'one-point interaction' is further consistent with the very small calculated energy difference between the two diastereomeric complexes, which is also in agreement with experiment. Still, even such weakly enantiodiscriminating interactions lead to detectable signals in the lute configuration of proline. The demodulated spectra can be viewed as difference spectra between the relevant diastereomeric complexes. The position of the dispersive bands suggests frequency shifts of the proline carboxylate $\mathrm{v}_{\mathrm{as}}\left(\mathrm{COO}^{-}\right)$(possibly also $\left.v_{\mathrm{s}}\left(\mathrm{COO}^{-}\right)\right)$and $\mathrm{NH}_{2}^{+}$scissoring $\delta\left(\mathrm{NH}_{2}{ }^{+}\right)$ and NAC amide and carboxylate vibrations (compare the spectra of NAC and proline at the bottom in Fig. 8).

Density functional theory (DFT) calculations of an NAC-proline complex were performed using GAUSSIAN03 [31] in order to further study the origin of the observed enantiodiscrimination using the hybrid functional B3PW91 with a 6-31G basis set. Solvent $(\mathrm{EtOH})$ effects were accounted for by performing optimizations using a polarizable continuum model (PCM). The conformational space of the complex was further probed by a Born-Oppenheimer molecular dynamics (BOMD) using the semi-empirical AM1 Hamiltonian. Among different NAC-proline intermolecular forces the ionic interaction between the positively charged proline $\mathrm{NH}_{2}{ }^{+}$ group and the negatively charged NAC carboxylate $\mathrm{COO}^{-}$turned out to dominate the interaction. Structure optimization was performed for the NAC-D-proline and NAC-Lproline diastereomeric complexes and a pictorial representation is given in Fig. 9. The difference in potential energy between the two chiral complexes was finally found to be only about $0.2 \mathrm{kJmol}^{-1}$.

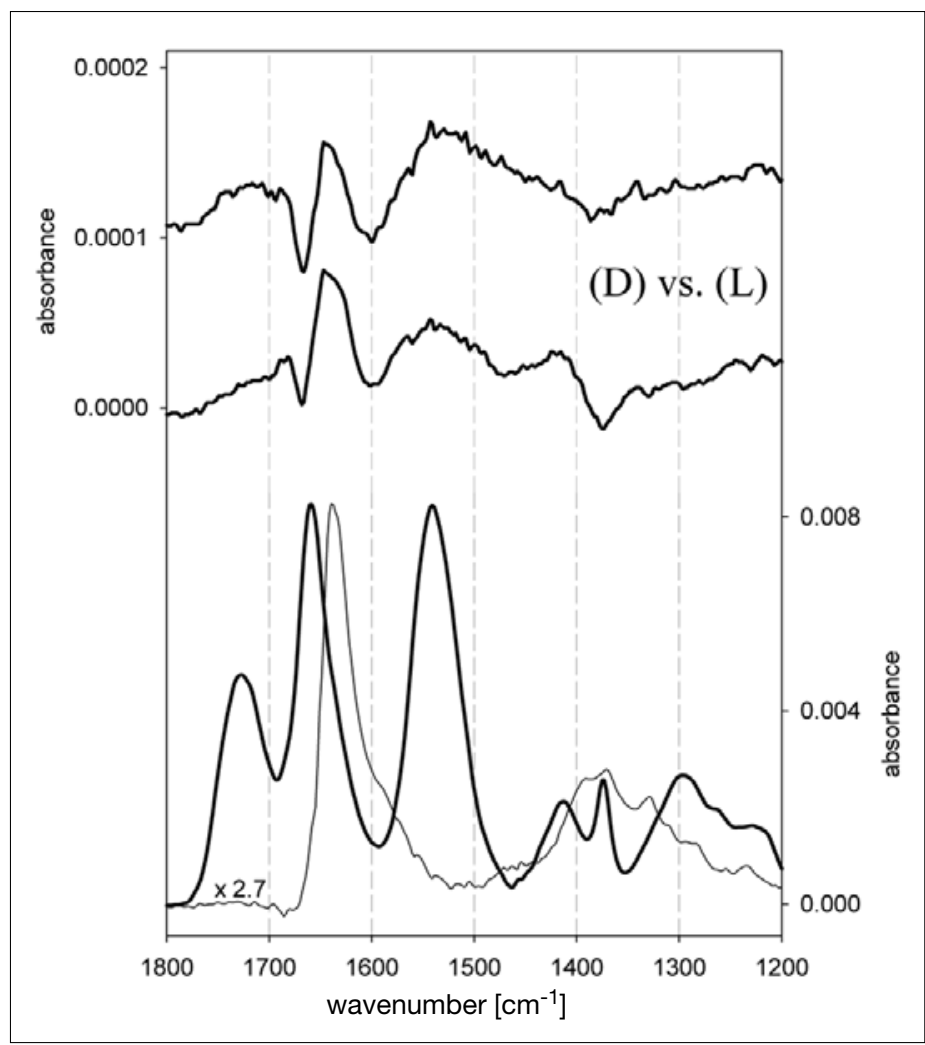

Fig 8. Bottom: ATR-IR spectra of N-acetyl-L-cysteine (NAC, selector, bold solid line) adsorbed on a gold surface and proline (selectand, thin solid line, scaled by a factor of 2.7) dissolved in ethanol. Top: demodulated, i.e. phase-resolved ATR-IR spectra. In this type of modulation experiment, D- and L-proline were periodically flowed over a chiral NAC SAM (see also Fig. 7). The two demodulated spectra highlight the signals arising due to enantiospecific interaction between NAC and proline. The two experiments were performed on different days using different gold surfaces illustrating the reproducibility of the absolute configuration modulation experiments. Reprinted with permission from [7]. 


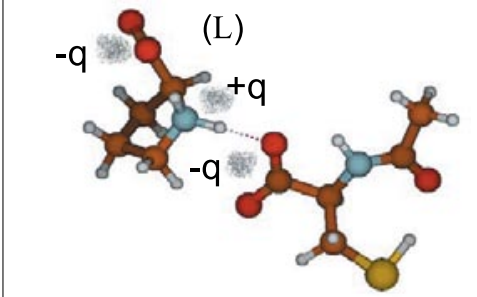

a)

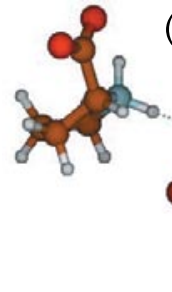

(D)

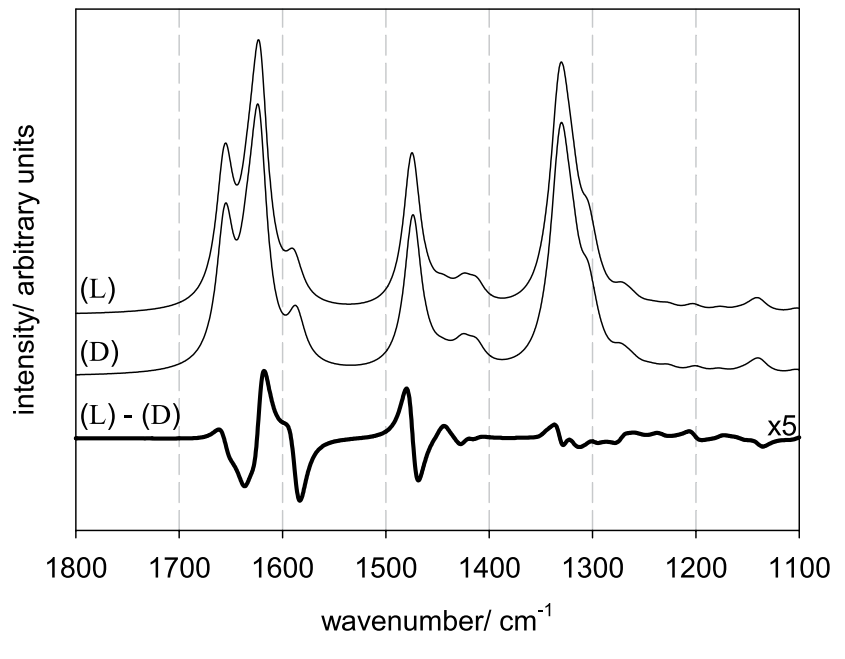

Fig. 9. Top: Pictorial representation of the optimized structures of the NAC-L-proline a) and NAC-L-proline b) complexes. The structure of the complexes is determined by ionic proline $\mathrm{NH}_{2}{ }^{+} \ldots \mathrm{COO}^{-} \mathrm{NAC}$ intermolecular interactions. In a) the charges are schematically indicated by clouds. Bottom: Calculated vibrational spectra of NAC-D-proline ('D') and NAC-Lproline ('L') complexes. The corresponding difference spectrum ' $(L)-(D)$ ', scaled by a factor of 5 , is presented at the bottom as bold solid line. Note that the calculated difference spectrum is experimentally accessible by ATR-IR and corresponds to the phase-resolved spectra on top in Fig. 8. Reprinted with permission from [7]

difference spectrum of the two diastereomeric complexes as revealed by absolute configuration modulation. This shows that the technique is a sensitive tool for the study of enantiodiscrimination at chiral solidliquid interfaces.

\section{Acknowledgments}

Financial support by the Swiss National Science Foundation and grants of computer time by the Swiss National Supercomputing Centre (Manno) are kindly acknowledged. We also thank Dr. M. Dadras for the TEM measurements.

Received: September 4, 2006

[1] C.F. McFadden, P.S. Cremer, A.J. Gellman, Langmuir 1996, 12, 2483.

[2] G.A. Attard, J. Phys. Chem. B 2001, 105, 3158.

[3] M.O. Lorenzo, C.J. Baddeley, C. Muryn, R. Raval, Nature 2000, 404, 376.

[4] V. Humblot, R. Raval, Appl. Surf. Sci. 2005, 241, 150.

[5] A.F. Carley, M.K. Rajumon, M.W. Roberts, P.B. Wells, J. Chem. Soc., Faraday Trans. 1995, 91, 2167.

[6] D. Ferri, T. Bürgi, J. Am. Chem. Soc. 2001, 123,12074
[7] M. Bieri, T. Bürgi, Chem. Phys. Chem. 2006, 7, 514.

[8] R.J. LeBlanc, W. Chu, C.T. Williams, $J$. Mol. Catal A: Chemical 2004, 212, 277.

[9] V. Humblot, S. Haq, C. Muryn, W.A. Hofer, R. Raval, J.Am. Chem. Soc. 2002, 124 , 503.

[10] W.A. Hofer, V. Humblot, R. Raval, Surf. Sci. 2004, 554, 141

[11] M.-C. Daniel, D. Astruc, Chem. Rev. 2004, 104, 293.

[12] J.A. Schellman, Chem. Rev. 1975, 75, 323.

[13] L.A. Nafie, T.A. Keiderling, P.J. Stephens, J. Am. Chem. Soc. 1976, 98, 2715.

[14] C. Gautier, T. Bürgi, J. Chem. Soc. Chem. Commun. 2005, 5393.

[15] M. Studer, H.U. Blaser, C. Exner, $A d v$. Synth. Catal. 2003, 345, 45.

[16] T. Bürgi, A. Baiker, Acc. Chem. Res. 2004, 37, 909.

[17] S. Ahuja, 'Chiral separations by chromatography', Oxford University Press, Washington, DC, 2000.

[18] R. McKendry, M.-E. Theoclitou, T. Rayment, C. Abell, Nature 1998, 391, 566.

[19] N.J. Harrick, 'Internal reflection spectroscopy', Interscience Publishers, New York, 1967.
[20] D. Baurecht, U.P. Fringeli, Rev. Sci. Instr 2001, 72, 3782.

[21] C. Gautier, T. Bürgi, J. Am. Chem. Soc. 2006, 128, 11079 .

[22] R. Di Felice, A. Selloni, E. Molinari, J. Phys. Chem. B 2003, 107, 1151.

[23] M. Bieri, T. Bürgi, J. Phys. Chem. B 2005 , 109, 22476.

[24] M.E. Marti, C. Methivier, C.M. Pradier, Langmuir 2004, 20, 10223.

[25] I.L. Garzon, J.A. Reyes-Nava, J.I. Rodriguez-Hernandez, I. Sigal, M.R. Beltran, K. Michaelian, Phys. Rev. B 2002, 66 73403.

[26] M.-R. Goldsmith, C.B. George, G. Zuber, R. Naaman, W.D.H.P. Wipf, D.N. Beratan, Phys. Chem. Chem. Phys. 2006, 8, 63.

[27] R. Kessinger, J. Crassous, A. Herrmann, M. Rüttimann, L. Echegoyen, F. Diederich, Angew. Chem, Int. Ed. 1998, 37, 1919.

[28] A. Herrmann, M. Rüttimann, C. Thilgen, F. Diederich, Helv. Chim. Acta 1995, 78 , 1673.

[29] R. Wirz, T. Bürgi, A. Baiker, Langmuir 2003, 19, 785 .

[30] R. Wirz, T. Bürgi, W. Lindner, A. Baiker, Anal. Chem. 2004, 76, 5319.

[31] M.J. Frisch, G.W. Trucks, H.B. Schlegel, G.E. Scuseria, M.A. Robb, J.R. Cheeseman, J.A. Montgomery, T. Vreven, K.N. Kudin, J.C. Burant, J.M. Millam, S.S. Iyengar, J. Tomasi, V. Barone, B. Mennucci, M. Cossi, G. Scalmani, N. Rega, G.A. Petersson, H. Nakatsuji, M. Hada, M. Ehara, K. Toyota, R. Fukuda, J. Hasegawa, M. Ishida, T. Nakajima, Y. Honda, O. Kitao, H. Nakai, M. Klene, X. Li, J.E. Knox, H.P. Hratchian, J.B. Cross, C. Adamo, J. Jaramillo, R. Gomperts, R.E. Stratmann, O. Yazyev, A.J. Austin, R. Cammi, C. Pomelli, J.W. Ochterski, P.Y. Ayala, K. Morokuma, G.A. Voth, P. Salvador, J.J. Dannenberg, V.G. Zakrzewski, S. Dapprich, A.D. Daniels, M.C. Strain, O. Farkas, D.K. Malick, A.D. Rabuck, K. Raghavachari, J.B. Foresman, J.V. Ortiz, Q. Cui, A.G. Baboul, S. Clifford, J. Cioslowski, B.B. Stefanov, G. Liu, A. Liashenko, P. Piskorz, I. Komaromi, R.L. Martin, D.J. Fox, T. Keith, M.A. Al-Laham, C.Y. Peng, A. Nanayakkara, M. Challacombe, P.M.W. Gill, B. Johnson, W. Chen, M.W. Wong, C. Gonzalez, J.A. Pople, Rev. C.01 ed., Gaussian, Inc., Wallingford CT, 2003. 